\title{
Inferential Current Treatment
}

National Cancer Institute

\section{Source}

National Cancer Institute. Inferential Current Treatment. NCI Thesaurus. Code C21030.

A non-invasive form of electroanalgesia, based on TENS, that uses the principle of amplitude modulation to decrease the discomfort of stimulating deeper tissues when using transcutaneously applied electrical current. It is often used in physiotherapy and rehabilitation settings. (White, Li, and Chiu) 